\title{
PARASITOSIS INTESTINAL, ANEMIA Y RENDIMIENTO ESCOLAR
}

\author{
INTESTINAL PARASITOSIS, ANEMIA AND SCHOOL PERFOMANCE \\ Jhony A. De La Cruz -Vargas 1,2,4, Erick J. Wetzel³, Jorge Cárdenas-Callirgos ${ }^{3}$, Stephanie Velasquez - Vila ${ }^{3}$, \\ Lucy E. Correa-López ${ }^{4}$
}

\begin{abstract}
RESUMEN
Introducción: La anemia en niños es un problema de salud pública en el Perú y en el mundo. Los niños son particularmente vulnerables a la anemia, por su elevada velocidad de crecimiento y altos requerimientos de hierro, presencia de parásitos, bajo peso al nacer y episodios frecuentes de infecciones diarreicas. El objetivo de la presente investigación fue evaluar los factores asociados a parasitosis gastrointestinal, anemia y desempeño escolar en niños del nivel primario de dos colegios de Pamplona Alta. Métodos: Se realizó un estudio cuantitativo, analítico y transversal, con un muestreo probabilístico de tipo aleatorio simple, de estudiantes $1^{\circ}-6^{\circ}$ grado de primaria de dos colegios de Pamplona Alta, Lima, Perú. Se aplicó una encuesta previo consentimiento informado de los padres de familia, se tomaron medidas antropométricas de los niños, muestras de sangre capilar, así como muestras de coproparasitológico directo y test de Graham. Se eligió el promedio general del último trimestre así como el promedio final de matemáticas del último trimestre como indicadores del rendimiento escolar. Resultados: Un total de 109 niños fueron incluidos. Mediante examen directo de muestras de heces y el test de Graham se diagnosticó tres tipos de enteroparásitos patógenos: Entamoeba coli (6.54\%), Giardia lamblia (10.28\%) y Enterobius vermicularis (7.62\%). Esto corresponde a una prevalencia global de $24.44 \%$. Se observó además un $25.93 \%$ de anemia en la población en estudio. Hallamos asociación significativa entre anemia $<12 \mathrm{~g} / \mathrm{dl}$ y rendimiento global (OR: $4.09 \mathrm{p}=0,047$ IC95\%=1. 01- 20,591), anemia $<11.5$ y rendimiento global (0R: 5.60 IC95\%: 1.19 - 27.14 p: 0.01), y el tener mascota y aprobación en matemáticas (OR: $2.55 \mathrm{p}=0.041$ IC $95 \%=1.026$ - 6.374). Conclusión: Uno de cada 4 niños presento anemia y parasitosis, la anemia estuvo asociada significativamente a mal rendimiento escolar. El tener mascota se asoció significativamente a aprobación en matemáticas. Futuros estudios son necesarios para confirmar y ampliar nuestros resultados.
\end{abstract}

Palabras clave: Enteroparásitos; Perú; Anemia; Pamplona; Rendimiento escolar. (fuente: DeCS BIREME)

\begin{abstract}
Introduction: Anemia in children is a public health problem in Peru and in the world. Children are particularly vulnerable to anemia, due to their high growth rate and high iron requirements, the presence of parasites, low birth weight and frequent episodes of diarrheal infections. The objective of the present investigation was to evaluate the factors associated with gastrointestinal parasitosis, anemia and school performance in primary school children from two schools in Pamplona Alta. Methods: A quantitative, analytical and cross-sectional study was carried out, with a simple random probabilistic sampling form sudents 1st-6th grade primary classrooms of two schools in Pamplona Alta, Lima, Peru. A survey was applied with the informed consent of the parents, anthropometric measurements of the children were taken, capillary blood samples, as well as coproparasitological samples and the Graham test. The general average of the last trimester was chosen as well as the final math average of the last quarter as indicators of school performance. Results: A total of 109 children were included. By direct examination of stool samples and the Graham test, three types of pathogenic enteroparasites were diagnosed: Entamoeba coli (6.54\%), Giardia lamblia (10.28\%) and Enterobius vermicularis (7.62\%). This corresponds to a global prevalence of $24.44 \%$. A $25.93 \%$ anemia was also observed in the study population. We found a significant association between anemia $<12 \mathrm{~g} / \mathrm{dl}$ and overall performance (OR: $4.09 \mathrm{p}=0.047$ IC95\% = 1.01-20.591), anemia <11.5 and overall performance (OR: 5.60 OR: 5.6 IC95\%: $1.19-27.14$ p: 0.01 ) and having a pet and approval in math (OR: $2.55 p=0.041$ IC $95 \%=1.026-6.374)$. Conclusion: One out of every 4 children presented anemia, one out of every 5 children presented parasitosis, anemia was significantly associated with poor school performance. Having a pet was significantly associated with math approval. Future studies are necessary to confirm and extend our results.
\end{abstract}

Key words: Enteroparasites; Perú; Anemia; Pamplona; School performace. (source: MeSH NLM)

\footnotetext{
Director del Instituto de Investigación en Ciencias Biomédicas.URP, Lima-Perú.

${ }^{2}$ Maestría en Investigación Clínica y Doctorado en Medicina.

${ }^{3}$ Global Health Initiative-Perú, Wabash College, Indiana. USA.

${ }_{4}^{4}$ Facultad de Medicina Humana, INICIB-URP, Lima-Perú.
}

Citar como: Jhony A. De La Cruz -Vargas, Erick J. Wetzel, Jorge Cárdenas-Callirgos, Stephanie Velasquez - Vila, Lucy E. Correa-López. Parasitosis intestinal, anemia y rendimiento escolar. [Artículo Original].2018;18(4):30-39. (Octubre 2018). DOI 10.25176/RFMH.v18.n4.1728

( ) Los autores. Este artículo es publicado por la Revista de la Facultad de Medicina Humana, Universidad Ricardo Palma. Este es un artículo de Open Access distribuido bajo los términos de la Licencia Creative Commons Atribución-NoComercial-Compartirlgual 4.0 Internacional.(http://creativecommons.org/licenses/by-nc-sa/4.0/), que permite el uso no comercial, distribución y reproducción en cualquier medio, siempre que la obra original sea debidamente citadas. Para uso comercial, por favor póngase en contacto con revista.medicina@urp.pe 


\section{INTRODUCCIÓN}

SSegún la Organización Mundial de la Salud' la anemia en niños es un problema de salud pública en el Perú y en el mundo. Los niños son particularmente vulnerables a la anemia, por su elevada velocidad de crecimiento y altos requerimientos de hierro, presencia de parásitos, bajo peso al nacer y episodios frecuentes de infecciones diarreicas. Diversos estudios han documentado como la anemia impacta negativamente en el desarrollo psicomotor, cognitivo, social y emocional. La anemia puede disminuir el desempeño escolar'.

El Perú es uno de los países más afectados por enfermedades transmisibles, y las parasitosis constituyen aún un problema de salud pública, ya que constituyen una de las diez primeras causas de muerte (Organización Panamericana de la Salud, 1998). Es probable que tres cuartos de la población peruana porte algún parásito intestinal', esta relación se hace más evidente de acuerdo a las características de la población, siendo mayor en la población rural y urbano marginal; y mayor en niños que en adultos ${ }^{2-5}$.

Una de las principales consecuencias de la parasitosis intestinal es la desnutrición crónica que produce, pudiendo desarrollar en algunos casos anemia. Esto hace que los niños, que son los principales afectados por este problema, no tengan un adecuado desarrollo físico e intelectual ${ }^{4,5}$.

El Perú presenta índices elevados de parasitosis intestinales en Latinoamérica, siendo las poblaciones de bajo nivel socioeconómico y los niños, las más afectadas ${ }^{6}$.

El objetivo del presente estudio fue determinar los factores asociados a parasitosis intestinal, anemia y rendimiento escolar en niños de Pamplona Alta, San Juan de Miraflores, Lima, Perú.

\section{MÉTODOS}

\section{Diseños de investigación}

El presente es un estudio cuantitativo, analítico, transversal.

\section{Población de estudio}

La población de estudio fueron los niños en edad escolar de $1^{\circ}-6^{\circ}$ grado de primaria, de dos colegios de Pamplona Alta, San Juan de Miraflores, Lima que se encontrarán cursando el segundo bimestre académico del año escolar.

Se realizó un tipo de muestreo probabilístico de tipo aleatorio simple. A partir de la lista de escolares de los salones de $1^{\circ}-6^{\circ}$ grado de primaria de dos colegios de Pamplona Alta, Lima, Perú.

\section{Instrumentos de recolección de datos}

Encuesta ficha de recoleccion de datos.

\section{Técnicas de procesamiento de datos}

En el ordenamiento de los datos se usará el programa Microsoft Excel (versión 2010 para Windows), luego se realizará el análisis con el programa estadístico Stata 11.1 (StataCorp LP, College Station, TX, USA). Las variables cuantitativas serán representadas por medidas de tendencia central (media o mediana) y medidas de dispersión (desviación estándar o rango intercuartílico), previa evaluación de la distribución de los valores. Para las variables cualitativas se utilizarán medidas de distribución de frecuencia.

Para la estadística analítica el nivel de confianza será de $95 \%$. Las variables categóricas se evaluarán con la prueba de chi cuadrado, que será corregida con la prueba de Fisher para frecuencias pequeñas. Las variables numéricas serán analizadas con $t$ de Student, corregida por la suma de rangos cuando no se cumpliera los criterios de normalidad. Los valores $p<0.05$ se considerará como estadísticamente significativos.

\section{Procedimientos}

Los datos fueron recolectados de los escolares de dos colegios de la localidad de Pamplona Alta, en el distrito de San Juan de Lurigancho, departamento de Lima, Perú; previa charla informativa a los niños y a los padres de familia, con orientación de educacion para la salud y preventiva; luego de lo cual, se aplicaron encuestas, las cuales fueron llenadas por los padres de familia, en las que se recolectaron las variables epidemiológicas de interés. Por otro lado, las variables clínicas fueron evaluadas por los médicos participantes del proyecto.

Para el análisis parasitario se recolectaron muestras de heces y se usó el Test de Graham que consiste en la toma de muestra mediante una cinta adhesiva que debe ser transparente (no solamente translúcida) para recolectar en la región anal y perianal cualquier huevo con el objeto de visualizarlos directa con un microscopio. Las muestras recolectadas fueron examinadas en el laboratorio realizándose examen directo, sedimentación y flotación por personal técnico calificado para así identificar la presencia de parásitos intestinales.

Se tomaron indicadores antropométricos: Índice de masa Corporal (IMC): Peso/Edad, Talla/Edad, Peso/Talla, para lo cual se utilizarán tablas antropométricas de la 
National Center for Health Stadistics. El rendimiento académico fue evaluado de acuerdo al calificativo anual.

Todos estos procedimientos fueron realizados previa firma del consentimiento informado por parte de los padres de familia y el asentimiento de los menores que tengan la edad suficiente para hacerlo. Además, el presente proyecto de investigación fue aprobado por la Facultad de Medicina Humana-INICIB, y el Vicerrectorado de Investigación.

\section{RESULTADOS}

Se incluyó en el estudio un total de 109 escolares del nivel primario de dos colegios de Pamplona Alta, en Lima-Peru, con características socioeconómicas similares, que cumplieron con la recolección de sus muestras y evaluaciones programadas. La edad promedio fue de 9 años \pm 1.79 y varió entre los 6 y 14 años de edad. El 55\% (60/109) fueron del sexo femenino y el 45\% (49/109) del sexo masculino, GRAFICO 1. Participaron del estudio alumnos de 1 a 6 grado de primaria, TABLA 1.

Tabla 1. Frecuencia de los alumnos según grado escolar.

\begin{tabular}{|lcc|}
\hline & FRECUENCIA & PORCENTAJE \\
\hline $1^{\circ}$ de primaria & $+/ N$ & 28.4 \\
\hline $2^{\circ}$ de primaria & 31 & 15.6 \\
\hline $3^{\circ}$ de primaria & 17 & 23.9 \\
\hline $4^{\circ}$ de primaria & 26 & 11.0 \\
\hline $5^{\circ}$ de primaria & 12 & 6.4 \\
\hline $6^{\circ}$ de primaria & 7 & 14.7 \\
\hline Total & 16 & 100.0 \\
\hline
\end{tabular}

La altura media fue de 1.32 metros +- 0.10 (1.12-1.60 m.). El peso medio fue de 34,99 kilogramos +- 10.17 (18.9070.50) y el Indice e Masa Muscular (IMC) mostró una media de 19.76+-3.51(13.50-32.19). La hemoglobina media fue de 12.52 mg. +- 0.74 (10.8-14.00).

Sobre 7 cursos por trimestre, se encontró un máximo de 3 cursos desaprobados en el primer trimestre y 2 cursos desaprobados en el segundo trimestre del 2017. Se evaluó rendimiento en matemáticas encontrándose un bajo rendimiento en el 19\% (20 alumnos).

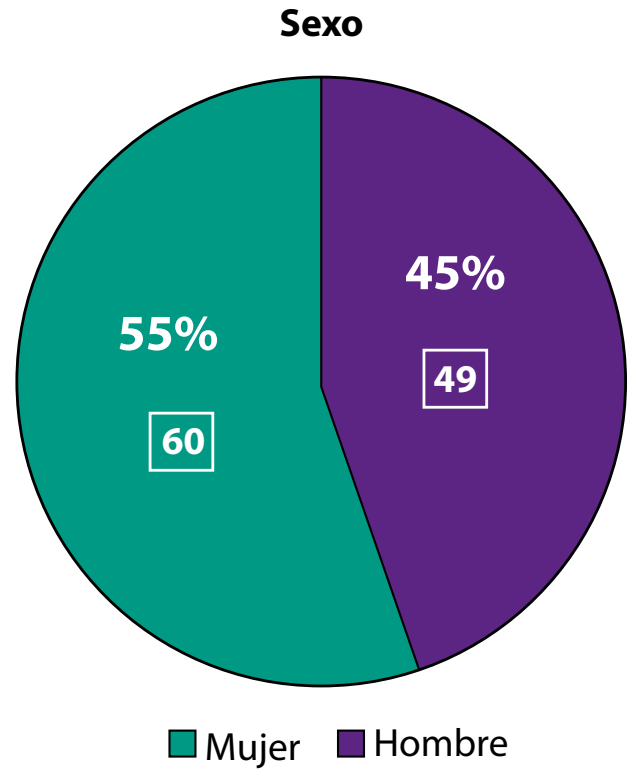

Gráfico 1. Distribución de los alumnos según sexo.

\section{Factores socioeconómicos}

Alrededor del $88 \%$ de los estudiantes contaban con al menos algún servicio básico en su hogar (agua, luz y/o desagüe) pero más de la mitad (50.5\%) no contaba con un sistema de desagüe para la eliminación de excretas. El servicio de recolección de basura, según respondieron los encuestados, alcanza al 95.4\% de los hogares. La cría de animales en el hogar resalta como una práctica común entre los hogares de los estudiantes de estos dos colegios de Pamplona (77.1\%, Tabla 2), ya sea animales domésticos o de corral. 
Tabla 2. Calidad de servicios sanitarios y cría de animales.

\begin{tabular}{|c|c|c|}
\hline & $\begin{array}{c}\text { FRECUENCIA } \\
+/ \mathbb{N}\end{array}$ & $\begin{array}{c}\text { PORCENTAJE } \\
\%\end{array}$ \\
\hline \multicolumn{3}{|l|}{ Servicios básicos } \\
\hline Agua & $68 / 109$ & 62.4 \\
\hline Desagüe & $59 / 109$ & 54.1 \\
\hline Luz & $76 / 109$ & 69.7 \\
\hline Ninguno & $30 / 109$ & 27.5 \\
\hline \multicolumn{3}{|l|}{ Eliminación de excretas en el hogar } \\
\hline Sistema de desagüe & $54 / 109$ & 49.5 \\
\hline Otro tipo de eliminación (letrina o campo abierto) & $55 / 109$ & 50.5 \\
\hline \multicolumn{3}{|l|}{ Forma de eliminación de la basura } \\
\hline Campo abierto & $4 / 109$ & 3.7 \\
\hline Quema & $1 / 109$ & 0.9 \\
\hline Camión recolector & $104 / 109$ & 95.4 \\
\hline \multicolumn{3}{|l|}{ Cría animales en casa } \\
\hline Sí (perro, gato, cerdo, cuy, conejo o ave de corral) & $84 / 109$ & 77.1 \\
\hline 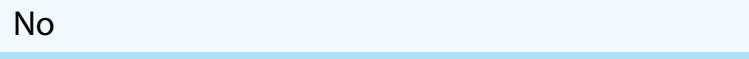 & $25 / 109$ & 22.9 \\
\hline \multicolumn{3}{|l|}{ Número de personas en el hogar (hacinamiento) } \\
\hline Menos de 5 & $35 / 109$ & 32.1 \\
\hline De 5 a 8 & $65 / 109$ & 59.6 \\
\hline De 9 a 12 & $3 / 109$ & 2.8 \\
\hline Más de 12 & $6 / 109$ & 5.5 \\
\hline
\end{tabular}

Más de 12

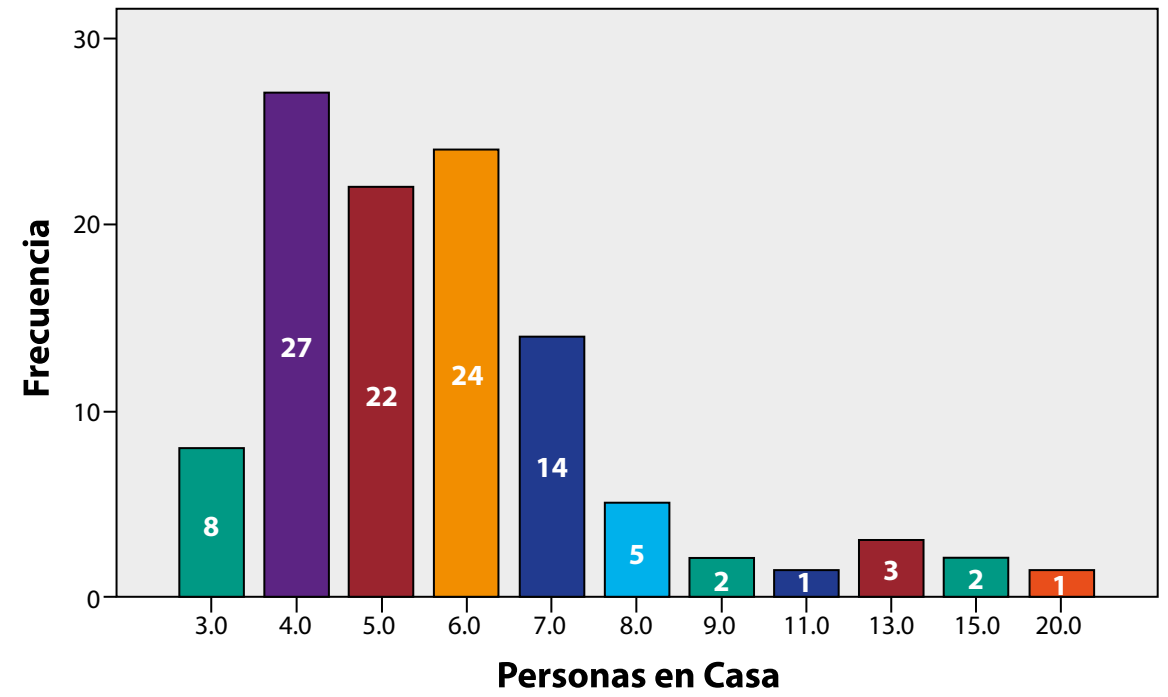

Gráfico 2. Distribución según número de Personas que comparten la vivienda. 


\section{Rendimiento escolar}

Se eligió el promedio general en el último trimestre así como el promedio final de matemáticas del último trimestre como indicadores del rendimiento, ambos se muestran en la Tabla 3.

Cuando agrupamos las calificaciones muy buenas AD (4 estudiantes) mas las calificaciones buenas A (94 estudiantes), tenemos el grupo de "alto rendimiento" (98 estudiantes) comparado con el grupo de calificaciones deficientes B (9 estudiantes) más calificaciones desaprobatorias C (2 estudiantes) de "bajo rendimiento" (11 estudiantes).

Según el promedio de matemáticas se dividió en "alto rendimiento" (AD+A: 89 estudiantes) y bajo rendimiento ( $B-C: 20$ estudiantes).

Tabla 3. Indicadores de rendimiento escolar del último trimestre.

\begin{tabular}{|c|c|c|}
\hline & $\begin{array}{c}\text { FRECUENCIA } \\
+/ N\end{array}$ & $\begin{array}{c}\text { PORCENTAJE } \\
\%\end{array}$ \\
\hline \multicolumn{3}{|c|}{ Promedio general } \\
\hline$A D(18$ a 20$)$ & $4 / 109$ & 3.7 \\
\hline A (14 a 17) & $94 / 109$ & 86.2 \\
\hline B (11 a 13) & 9/109 & 8.3 \\
\hline$C(0$ a 10$)$ & $2 / 109$ & 1.8 \\
\hline \multicolumn{3}{|c|}{ Promedio en matemáticas (Correspondencia numérica) } \\
\hline$A D(18$ a 20$)$ & $5 / 109$ & 4.6 \\
\hline$A(14$ a 17) & 847109 & 77.1 \\
\hline B (11 a 13) & $18 / 109$ & 16.5 \\
\hline$C(0$ a 10$)$ & $2 / 109$ & 1.8 \\
\hline
\end{tabular}

\section{Parasitosis}

Se encontró una prevalencia de $16.82 \%$ (17/107) de parasitosis gastrointestinal en el coproparasitologico directo. Las prevalencias para cada tipo de parásito encontrado se observan en la Tabla 4. El hallazgo del protozoario Endolimax nana en las muestras no fue considerado como infección parasitaria por tratarse de un parásito no patógeno. De los 109 estudiantes incluidos en el estudio, 107 entregaron muestras de heces válidas para el descarte de parásitos mediante muestras directas de heces. Se observó presencia de 2 especies de protozooarios patógenos: Entamoeba coli (6.54\%) y Gardia lamblia (10.28\%). De los 109 estudiantes incluidos en el estudio, 105 entregaron el test de Graham para el descarte de huevos de Enterobius vermicularis u oxiuros. Más del siete por ciento $(7.62 \%)$ de los estudiantes evaluados presentó huevos de oxiuros. Dependiendo de la carga de huevos de oxiuros por campo en el test, el laboratorio dio tres grados diferentes de infección.

\section{Prevalencia de Parasitosis}

La prevalencia de parasitosis por examen directo de coprocultivo en los niños estudiados fue de $16.82 \%$ (17 niños), de los cuales 11 escolares fueron positivos para Giardia lamblia. Adicionalmente la prevalencia de parasitosis por test de Graham para Oxiuro fue de $7.62 \%$ positivos, dando una prevalencia global de $24.44 \%$. Entamoeba coli (6.54\%), Giardia lamblia (10.28\%) y Enterobius vermicularis (7.62\%).

\section{Anemia}

El valor medio de hemoglobina en los niños fue de 12.52 $\pm 0.74 \mathrm{~g} / \mathrm{dl}$ oscilando en un rango entre $10.8-14.0 \mathrm{~g} / \mathrm{dl}$ (Tabla 1). De los 109 estudiantes incluidos en el estudio, 108 pudieron ser muestreados para la evaluación de concentración de hemoglobina. Usando $12 \mathrm{~g} / \mathrm{dl}$ de hemoglobina como punto de corte, se observó que la prevalencia de anemia fue de $25.9 \%$ ( 28 niños), y una prevalencia de $8.3 \%$ (9 niños) para hemoglobina menor a $11.5 \mathrm{mg} \%$.

\section{Estado Nutricional}

En cuanto al estado nutricional, el $66.1 \%$ de los niños mostro estar hipotrofico, solo el $26.6 \%$ presentaba rango normal. El $7.5 \%$ de los niños correspondieron a exceso de peso: sobrepeso (6.4\%) y obesidad (0.9\%).

\section{Promedio por trimestre}

Si agrupamos las calificaciones en promedio por trimestre en $A+A D$, el $89.9 \%$ obtuvo calificaciones satisfactorias y muy satisfactorias. El 10\% (11 niños) obtuvieron calificaciones B y C. Al evaluar su desempeño según su calificación en promedio de matemáticas, encontramos que el $81 \%$ correspondió a $A+A D$, y un $19 \%$ ( 20 alumnos) a $B+C$.

\section{ASOCIACIÓN DE VARIABLES}

Las tablas 5 y 6 muestran los factores asociados por analisis bivariable. 
Tabla 4. Indicadores de rendimiento escolar del último trimestre.

\begin{tabular}{|c|c|c|}
\hline & $\begin{array}{c}\text { FRECUENCIA } \\
+/ N\end{array}$ & $\begin{array}{c}\text { PORCENTAJE } \\
\%\end{array}$ \\
\hline \multicolumn{3}{|l|}{ Parasitosis gastrointestinal } \\
\hline No se observaron formas parasitarias & $90 / 107$ & 84.11 \\
\hline Q. de Entamoeba coli & $7 / 107$ & 6.54 \\
\hline Q. de Giardia lamblia & $11 / 107$ & 10.28 \\
\hline \multicolumn{3}{|l|}{ Huevos de oxiuros } \\
\hline Bajo & $3 / 105$ & 2.86 \\
\hline Medio & $4 / 105$ & 3.81 \\
\hline Alto & $1 / 105$ & 0.95 \\
\hline \multicolumn{3}{|l|}{ Anemia } \\
\hline Anémicos $(\mathrm{Hb}<12.0 \mathrm{~g} / \mathrm{dl})$ & $28 / 108$ & 25.93 \\
\hline Anemicos ( $\mathrm{Hb}<11.5 \mathrm{~g} / \mathrm{dl})$ & 09/108 & 8.30 \\
\hline \multicolumn{3}{|l|}{ Estado Nutricional } \\
\hline Hipotrófico & $72 / 109$ & 66.1 \\
\hline Normal & 29/109 & 26.6 \\
\hline Sobrepeso & $7 / 109$ & 6.4 \\
\hline Obesidad & $1 / 109$ & 0.9 \\
\hline
\end{tabular}

Tabla 5. Factores Asociados a Bajo Rendimiento según Promedio Global del Ultimo Trimestre.

\begin{tabular}{|lccc|}
\hline \multicolumn{1}{|c}{ VARIABLE } & OR & IC95\% & P \\
\hline Anemia menor de 12 ( SI/NO) & 4.09 & $1.01-20.59$ & 0.047 \\
\hline Anemia menor de 11.5 ( SI/NO) & 5.60 & $1.19-27.14$ & 0.01 \\
\hline Parasitosis (SI/NO) & 1.23 & $0.24-6.26$ & 0.80 \\
\hline Exceso de peso (SI/NO) & 1.30 & $0.14-11.67$ & 0.81 \\
\hline
\end{tabular}

Tabla 6. Factores Asociados a Desaprobación en Matemáticas del Ultimo Trimestre.

\begin{tabular}{lccc}
\hline \multicolumn{1}{c}{ VARIABLE } & OR & IC95\% & P \\
\hline $\begin{array}{l}\text { hipotrofia o bajo peso/ } \\
\text { sobrepeso+obesidad }\end{array}$ & 1.17 & $0.50-2.72$ & 0.70 \\
\hline $\begin{array}{l}\text { parasitosis (Si/NO) } \\
\text { Tener mascota (SI/NO) }\end{array}$ & 1.02 & $0.33-2.88$ & 0.96 \\
\hline Anemia menor de 12 ( SI/NO) & 2.55 & $1.02-6.37$ & 0.04 \\
\hline Anemia menor de 11.5 (SI/NO) & 1.3 & $0.32-1.91$ & 0.59 \\
\hline
\end{tabular}

No se encontró asociación significativa entre la presencia de parasitosis y anemia. 


\section{DISCUSIÓN}

Nuestro estudio muestra el perfil de los alumnos de primaria en Pamplona Alta, distrito de San Juan de Miraflores, Lima, Perú que comparten características socioeconómicas similares en relación a los factores asociados a parasitosis, anemia y rendimiento escolar.

Dentro de las características generales estudiadas, la vivienda, se resalta que el $37.6 \%$ no contaba con agua potable en su casa, $45.9 \%$ no contaba con desagüe y un $30.3 \%$ no contaba con servicio eléctrico. Asimismo, el $77.1 \%$ de los alumnos refirió tener mascotas o animales en su vivienda. La distribución por sexo es balanceada, Femenino: Masculino F: M 1.2 con un leve predominio femenino.

En cuanto al Índice de Masa Muscular (IMC), si bien se halló una media de 19.76+-3.51(13.50-32.19), resulta llamativo ver que solo el $26.6 \%$ de los niños tenían un IMC normal, encontrándose por un lado que el $66.1 \%$ de los alumnos tenían IMC por debajo de lo normal (hipotrófico), y por otro lado solo el $7.3 \%$ con sobrepeso y obesidad. Este perfil con claro predominio de bajo IMC, baja frecuencia de sobrepeso (6.4\%) y escasa obesidad $(0.9 \%)$, difiere de la media nacional y las tendencias de sobrepeso y obesidad en Perú y Latinomaerica ${ }^{21}$. El estado nutricional de los estudiantes evaluados es bajo en comparación con el observado en otro estudio realizado en el mismo distrito" ${ }^{11}$. Esto podría deberse a un cambio sustancial en las condiciones socioeconómicas del distrito en los últimos años, así como a la inequidad de los distritos más pobres de Lima, siendo Pamplona Alta uno de los sectores más empobrecidos en comparación con los otros sectores de San Juan de Miraflores. Un indicador relevante es la poca presencia de sistemas de desagües en las casas (Tabla 2). Este último reflejando el riesgo potencial para enfermedades parasitarias y de salud pública.

El análisis de muestras para descarte de parásitos gastrointestinales en 107 niños en edad escolar del nivel primario de Pamplona Alta, Lima, Perú, indican una exposición de al menos el $24.44 \%$ de los niños a contaminación fecal que representa un riesgo de infección. Esta prevalencia es menor a la observada en otros estudios en niños de San Juan de Miraflores y otros distritos de la ciudad de Lima y otras regiones del Perú (ver Tabla 5). Esta baja prevalencia reportada podría estar relacionada a que el examen directo realizado es menos sensible que los métodos de concentración que se suelen utilizar en otros estudios, por un lado y al número de muestras recibidas por cada niño estudiado.

En nuestro estudio, se observó un predominio de la presencia de protozoarios sobre los helmintos, esto podría deberse, como es resaltado en otros estudios realizados en Lima ${ }^{9}$, a factores ambientales que promueven una prevalencia mayor de este tipo de parásitos en la costa del Perú.

Las prevalencias de anemia observadas en Jamaica ${ }^{18}$ (14.7\%) y Brasil ${ }^{12}(26.7 \%)$ fueron similares a la observada para este estudio (25.93\%) lo que concuerda también con la prevalencia estimada de anemia para niños entre 6 y 12 año en Sudamérica (24\%) ${ }^{23}$. Sin embargo, los autores piensan que la prevalencia encontrada representa un subregistro, posiblemente aplicado por el tamaño de la muestra y los métodos solo directos utilizados.

Un hallazgo interesante de nuestro estudio, es la asociación significativa que hayamos entre tener una mascota en la casa y el rendimiento escolar. Existe evidencia científica sobre esta asociación entre la tenencia de animales de compañía y el desempeño en la escuela y el aprendizaje. Un reciente artículo de revisión sistemática sintetiza algunos de los hallazgos más interesantes en este campo de estudio ${ }^{24}$. Un estudio que respondía a la pregunta "¿Qué pueden los niños aprender de y con sus mascotas: el ejemplo del perro y el gato?"en niños en edad preescolar, encontró usando una herramienta cualitativa que las mascotas proporcionan experiencias positivas y bienestar. Los animales proveían a los niños de nuevas experiencias y fomentaban el aprendizaje en subcategorías como"ser bueno en tareas relacionadas a la escuela"25. Otro estudio enfocado en estudiar la vinculación niño-mascota encontró en niños entre los 3 y 6 años una relación entre niveles altos de inteligencia verbal en niños moderadamente vinculados a sus mascotas en comparación con niños que no tenían una mascota en casa $^{26}$.

La asociación de la anemia y el rendimiento escolar es unos de los más importantes retos médicos de la actualidad $^{27}$. Nuestro estudio muestra una asociación significativa (OR: 4,09) entre anemia menor de 12 (SI/NO) y bajo rendimiento escolar (SI/NO) según las calificaciones bajas $(B+C)$ y altas $(A+A D)$. Dicha asociación aumenta (OR: 5.60) cuando se evaluó la anemia menor a 11.5 (SI/NO) asociado al bajo rendimiento escolar ( $\mathrm{SI} / \mathrm{NO})$ según las calificaciones bajas $(B+C)$ y altas $(A+A D)$. Indicando que los alumnos con anemia, tienen mayor riesgo de bajo rendimiento académico global.

La anemia en Perú es un tema de alta prioridad, $y$ en niños escolares, resulta necesario desarrollar programas estratégicos para revertir y corregir los bajos niveles de hemoglobina, ya que su impacto en el desarrollo de los niños, y sus consecuencias en el rendimiento escolar en el presente, así como el futuro desarrollo económico y social de los mismos, hace que el circulo vicioso de pobreza, subdesarrollo y enfermedad, se perpetúe. 
Tabla 7. Estudios de prevalencia de parasitosis realizados en el Perú en los últimos años (adaptada de Larrea et al. 2011)22

\begin{tabular}{|c|c|c|c|c|c|c|}
\hline $\begin{array}{l}\text { PRIMER AUTOR } \\
\text { (AÑO) }\end{array}$ & DISTRITO & LUGAR & EDAD & $\mathbf{N}^{\circ}$ & PREVALENCIA & $\begin{array}{c}\text { ESPECIES } \\
\text { ENCONTRADAS }\end{array}$ \\
\hline Rua O. (2010) & Llama & Cajamarca & $\begin{array}{c}1-4 \\
\text { primaria }\end{array}$ & 88 & 80.7 & $\begin{array}{l}\text { B. hominis } 61.4 \% \text {, E. coli } \\
30.7 \% \text {, G. lambdia } 9.1 \% \text {, } \\
\text { E. nana } 5.7 \% \text {, H. nana } \\
3.4 \% \text {, I. butchlii } 13.6 \% \text {, } \\
\text { E. vermicularis } 3.4 \% \text {, A. } \\
\text { lumbricoides } 1.1 \% \text {, Ch. } \\
\text { mesnili } 1.1 \% \text {. }\end{array}$ \\
\hline
\end{tabular}

$\begin{array}{llllll}\begin{array}{l}\text { Rodríguez Ulloa C. } \\ \text { (2010) }\end{array} \text { Baños del Inca } & \text { Cajamarca } & 0-12 & 41 & 90.0 & \begin{array}{l}\text { Ch. mesnili, E. nana, E. coli, } \\ \text { l. butchlii, G. lambdia, A. } \\ \text { lumbricoides, H. nana. }\end{array}\end{array}$

$\begin{array}{llll}\text { Vera D. (2010) San Martín Lima } \quad 3 \text { a } 5 & 64 & 57.8 \quad \begin{array}{l}\text { B. hominis } 35.9 \%, \\ \text { G. lambdia } 21.8 \% y \\ \text { enterobiosis } 18.7 \% .\end{array}\end{array}$

\begin{tabular}{|c|c|c|c|c|c|c|}
\hline Borjas P. (2009) & Comas & Lima & 5 a 16 & 61 & 85.2 & $\begin{array}{l}\text { B. hominis } 67.3 \% \text {, E. coli } \\
57.6 \% \text {, I. butchlii } 25 \% \text {, E. } \\
\text { vermicularis } 36.54 \% \text {, G. } \\
\text { lambdia } 32.69 \% \text {, H. nana } \\
30.57 \% \text {, E. histolytica/ } \\
\text { hartmanni } 19.23 \% \text {. }\end{array}$ \\
\hline $\begin{array}{l}\text { Rivera-Jacinto } \\
(2008)\end{array}$ & NC & Cajamarca & 1 a 4 & 47 & 48.9 & $\begin{array}{l}\text { G. lamblia } 39.1 \% \text { y A. } \\
\text { lumbricoides } 21.7 \% \text {; E. coli } \\
47.8 \% \text { y Ch. mesnili } 21.7 \% \text {. }\end{array}$ \\
\hline
\end{tabular}

Ubillus G. (2008)

San Juan de Miraflores

Lima

1 a 4

112

82

\begin{tabular}{|c|c|c|c|c|c|c|}
\hline $\begin{array}{l}\text { lannacone J. } \\
(2007)\end{array}$ & $\begin{array}{l}\text { Chorrillos y } \\
\text { Pachacamac }\end{array}$ & Lima & 4 a 13 & 162 & 72.2 & $\begin{array}{l}\text { E. vermicularis (31.9\%; } \\
45.5 \%), \text { A. lumbricoides } \\
(15.3 \% ; 35.5 \%), \text { T. trichura } \\
(9.7 \% ; 6.6 \%) \text {, H. nana } \\
(37.5 \% ; 8.8 \%) .\end{array}$ \\
\hline
\end{tabular}

E. vermicularis $66 \%$, $\mathrm{G}$. lamblia $17 \%$, B. hominis

$11 \%$, Heminolepis nana $3 \%$,

E. coli $3 \%$.
Calderón E. (2006)

San Miguel

Lima

4 a 18

190

65.3
G. lamblia 19.47, E. vermicularis $14.21 \%$; comensales E. coli, $36.32 \%$ y E. nana $44.74 \%$. lannacone J. (2006)

Surco

Lima primaria
192

54.7
E. coli 22.9\%, E. nana 19 . $3 \%, B$. hominis $12.5 \%$, E. vermicularis $10.4 \%$. 
Las intervenciones a nivel de anemia y deficiencia de hierro, que debemos desarrollar en nuestros escolares deben ser iniciadas a muy corto y urgente plazo, sin perder de vista que deben iniciarse paralelamente programas multidisciplinarios a mediano y largo plazo destinados a la modificación de la realidad psicosocial y económica adversa que rodea a la mayoría de estos niños. Los indicadores hematológicos que hemos valorado reflejan una parte causal importante de la problemática del rendimiento escolar deficiente inseparable de los factores ambientales y psicosociales negativos. Para esto es fundamental la participación de todas las instancias de la sociedad, partiendo de los estamentos centrales de gobierno, de las instituciones científicas y universidades, sociedades médicas y organizaciones civiles destinadas a contribuir al diagnóstico de la situación nutricional y de salud, y también de manera multidisciplinaria de la comunidad educativa (maestros, padres de familia y escolares) cuyo rol es fundamental en la ejecución de los planes ${ }^{27}$.

Completado el diagnóstico y los resultados, el equipo de investigación, regresó a las dos escuelas, para proveer información personalizada de los resultados a los padres de familia, indicarles medidas higienicodieteticas pertinentes, proveerles de tratamiento antiparasitario especifico al niño y la familia, así como un programa de seguimiento con los sistemas de salud locales. Se tuvo la oportunidad de compartir los resultados con los tomadores de decisiones de dichos colegios, profesores y con la Dirección y sus equipos de trabajo correspondientes. La recepción de los resultados fue motivadora y las madres de los niños mostraron su reconocimiento al equipo de investigación. Se alcanzaron los objetivos de sensibilizar a los padres de familia y docentes sobre la importancia de los factores estudiados y el rendimiento escolar.

No hallamos asociación significativa entre los otros indicadores de rendimiento escolar utilizados $y$ diferentes potenciales factores de riesgo, esto podría deberse al tamaño de la muestra así como a la existencia de otros factores que no fueron considerados, factores de interferencia difíciles de controlar, por ejemplo, niños que teniendo rendimiento escolar bajo no tuvieran, a la vez, problemas psicológicos, orgánicos, emocionales o cuyos hogares no hubiesen sido afectados temporal o definitivamente por la crisis económica o social del país. Las circunstancias sociales, económicas y ambientales que rodearon a la mayoría de los niños estudiados permitieron percibir que solo un bajo porcentaje fueran considerados como niños de "hogares normales" ${ }^{27,28}$.

Las principales limitaciones de nuestro estudios fueron, la dificultad de que todos los niños y sus padres participen del estudio, y cumplan con traer sus muestras de coproparasitologico y que los niños concurran a la extracción de sus muestras hematologicas, esto influyó en el tamaño de la muestra, que es una limitante. El diseño transversal, solo nos permite estudiar de manera concomitamte las variables, sin posible relación causal.

Futuras evaluaciones multicentricas, longitudinales, son necesarias, que confirmen y amplíen nuestros resultados, evalúen el impacto y evolución de la anemia, parasitosis, y rendimiento escolar, y se desarrollen programas transversales por parte de los tomadores de decisiones que permitan abordar la verdadera raíz de esta problemática relacionada con los determinantes socioeconómicos y educativos de dicha población.

\section{CONCLUSIÓN}

- La prevalencia global de parasitosis hallada por métodos directos fue de $\mathbf{2 4 . 4 4 \%}$ en niños del nivel primario de dos colegios de Pamplona Alta. (Uno de cada cuatro niños de primaria presento parasitosis).

- La prevalencia de anemia hallado fue de $25.93 \%$ (Uno de cada 4 niños del nivel primario, presentó anemia.)

- Se encontró asociación significativa entre anemia $<12$ y bajo rendimiento global en niños del nivel primario de dos colegios de Pamplona Alta.

- Se encontró asociación significativa entre anemia $<11.5$ y bajo rendimiento global.

- Se encontró asociación significativa entre tener mascota y mayor probabilidad de aprobar matemáticas.

Contribuciones de autoría: Los autores participaron en la generación, redacción y aprobación final del artículo original.

Financiamiento: El proyecto de investigación fue financiado por un grant de investigación de la Universidad Ricardo Palma.

Conflicto de interés: Los autores declaran no tener conflicto de interés en la publicación de este artículo.

Recibido: 20 de julio del 2018

Aprobado: 10 de Setiembre del 2018

Correspondencia: Jhony A. De La Cruz Vargas

Dirección: INICIB, Facultad de Medicina Humana, Edificio I-208. 2do piso. Avenida Benavides 5440, Surco, Lima-Perú.

Teléfono: 708-0000 / Anexo: 6016

Correo:jhony.delacruz@urp.edu.pe 


\section{REFERENCIAS BIBLIOGRÁFICAS}

1. Náquira C. Parasitosis II: Diagnóstico y tratamiento de las enteroparasitosis. 1997 [citado 5 de febrero de 2018]; Disponible en: https://www.scienceopen.com/document?vid=d9b87f64-6fe8-43b39ae7-f28252d16d06

2. Devera R, Cermeño JR, Blanco Y, Morales B, Cecilia M, Guerra X, et al. Prevalencia de blastocistosis y otras parasitosis intestinales en una comunidad rural del Estado Anzoátegui, Venezuela. Parasitol Latinoam. Julio de 2003;58 (3-4):95-100.

3. Jacinto E, Aponte E, Arrunátegui-Correa V. Prevalencia de parásitos intestinales en niños de diferentes niveles de educación del distrito de San Marcos, Ancash, Perú. Rev Medica Hered. 21 de diciembre de 2012;23 (4):235.

4. Marcos L, Maco V, Samalvides F, Terashima A, Espinoza JR, Gotuzzo E. Risk factors for Fasciola hepatica infection in children: a case-control study. Trans R Soc Trop Med Hyg. 1 de febrero de 2006;100(2):158-66.

5. Mendoza N, Berné $Y$, Moreno JM, Papalé-Centofanti J, Castro M, Torres $\mathrm{M}$, et al. Prevalencia de anemia, deficiencia de hierro y parasitosis intestinal y su relación con las condiciones socioeconómicas, en menores de 15 años de la comunidad rural Las Bucarita, Estado Lara, Venezuela. Rev Inst Nac Hig Rafael Rangel. Diciembre de 2014;45(2):64-78.

6. Naquira C. Las zoonosis parasitarias: problema de salud pública en el Perú. Rev Peru Med Exp Salud Pública. Diciembre de 2010;27:494-7.

7. Pajuelo Camacho G, Lujan Roca D, Paredes Perez B. Estudio de enteroparásitos en el Hospital de Emergencias Pediátricas, Lima-Perú. Rev Medica Hered. Julio de 2005;16(3):178-83.

8. Alarcón M, lannacone J, Blanco YE. Parasitosis intestinal, factores de riesgo y seroprevalencia de toxocariosis en pobladores del parque industrial de Huaycán, Lima, Perú. Neotropical Helminthol. 2010;4(1):17-36.

9. lannacone J, Benites MJ, Chirinos L. Prevalencia de infección por parásitos intestinales en escolares de primaria de Santiago de Surco Lima, Perú. Parasitol Latinoam. junio de 2006;61(1-2):54-62.

10. Ziegelbauer K, Steinmann $P$, Zhou H, Du Z-W, Jiang J-Y, Fürst T, et al. Self-rated quality of life and school performance in relation to helminth infections: case study from Yunnan, People's Republic of China. Parasit Vectors. 23 de julio de 2010;3:61.

11. Jiménez J, Vergel K, Velásquez-García SM, Vega F, Uscata R, Romero $S$, et al. Parasitosis en niños en edad escolar: relación con el grado de nutrición y aprendizaje. Horiz Méd. 2011;11(2):65-9.

12. Tsuyuoka R, Bailey JW, Guimarães AM d'Avila N, Gurgel RQ, Cuevas LE. Anemia and intestinal parasitic infections in primary school students in Aracaju, Sergipe, Brazil. Cad Saúde Pública. abril de 1999;15(2):413-21.

13. Casapía M, Joseph SA, Núñez C, Rahme E, Gyorkos TW. Parasite risk factors for stunting in grade 5 students in a community of extreme poverty in Peru. Int J Parasitol. junio de 2006;36(7):741-7.

14. Navarro RE. El rendimiento académico: concepto, investigación y desarrollo. REICE Rev Iberoam Sobre Calid Efic Cambio En Educ [Internet] 2 de julio de 2016 [citado 26 de febrero de 2018];1(2). Disponible en: https://revistas.uam.es/index.php/reice/article/view/5354

15. Santander OAE. El rendimiento académico, un fenómeno de múltiples relaciones y complejidades. Rev Vanguard Psicológica Clínica Teórica Práctica. 2011;2(2):144-73.
16. Cueto S, Guerrero G, León J, Zapata M, Freire S. ¿La cuna marca las oportunidades y el rendimiento educativo? Una mirada al caso peruano. Lima: GRADE; 2013 p. 102.

17. Román Y, Rodríguez Y, Gutierrez E, Aparco JP, Sánchez-Gómez I, Fiestas F. Anemia en la población infantil del Perú: aspectos clave para su afronte. Lima: CENAN: 2015

18. Hutchinson SE, Powell CA, Walker SP, Chang SM, Grantham-McGregor SM. Nutrition, anaemia, geohelminth infection and school achievement in rural Jamaican primary school children. Eur J Clin Nutr. noviembre de 1997;51(11):729-35.

19. Simeon D, Callender J, Wong M, Grantham-McGregor S, Ramdath DD. School performance, nutritional status and trichuriasis in Jamaican schoolchildren. Acta Paediatr Oslo Nor 1992. noviembre de 1994;83(11):1188-93.

20. Acuña Vilela G, Apaza Nina J, García Ccallocunto C, Medina Umpire L. Planeamiento Estratégico del Distrito de San Juan de Miraflores [Internet]. 2013 [citado 26 de febrero de 2018]. Disponible en: http://tesis.pucp.edu. pe/repositorio//handle/123456789/4547

21. Perú: Encuesta Demográfica y de Salud Familiar 2016 - Nacional y Regional [Internet]. Perú: INEl; 2016. Disponible en: https://www.inei.gob. pe/media/MenuRecursivo/publicaciones digitales/Est/Lib1433/index.html 22. Larrea N, Torres F, Tello M, Gutierrez E. Efecto de la administración de albendazol en los niveles de hemoglobina de niños entre ocho y doce años con enterobiasis intestinal. Rev Peru Epidemiol. 2011;15(1):61-4.

23. DeMaeyer E, Adiels-Tegman M. The prevalence of anaemia in the world World Health Stat Q Rapp Trimest Stat Sanit Mond. 1985;38(3):302-16.

24. Purewal R, Christley R, Kordas K, Joinson C, Meints K, Gee N, et al. Companion Animals and Child/Adolescent Development: A Systematic Review of the Evidence. Int J Environ Res Public Health. 27 de 2017;14(3). 25. Svensson AS. The impact of the animals on children\&\#039;s learning and their development - a study of what children learn from and with pets: the example of dog and cat. Probl Educ 21st Century. 2014;59:Discontinuous.

26. Poresky RH, Hendrix C. Companion Animal Bonding, Children's Home Environments, and Young Children's Social Development. Abril de 1989 [citado 16 de febrero de 2018]; Disponible en: https://eric. ed.gov/?id=ED312087

27. Soto R, Vega L, Mormontoy W, Madrid M, Escudero E, Vega R Y et al. Influencia de la Anemia y la Deficiencia de Hierro en e Rendimiento Escolar. Anales de Salud Mental (1993) IX: 35-48.

28. Ricciuti HM. (1983) Efectos de los Factores Ambientales y Nutriciona1es Adversos sobre e1 Desarrollo Mental. Ambiente. Nutrtcton y De- sarrollo Mental. pp: 1-25. Publica- ciones OPS/ OMS N2 450. Washing- ton DC

29. Idjradinata P \& Pollit E. (1993) Reversal of developmental delays in iron-deficien $t$ anaemic infants treated with iron. Lancet 341:1-4

30. World Health Organization. Guideline: Intermittent iron supplementation in preschool and school-age children. Geneva: $\mathrm{WHO}_{\text {; }}$ 2011. Disponible en: https://www.ncbi.nlm.nih.gov/books/ NBK179850/ 